\title{
Diffusion-Weighted Imaging Tractography-Based Parcellation of the Human Lateral Premotor Cortex Identifies Dorsal and Ventral Subregions with Anatomical and Functional Specializations
}

\author{
Valentina Tomassini, ${ }^{1,3}$ Saad Jbabdi, ${ }^{1}$ Johannes C. Klein, ${ }^{1}$ Timothy E. J. Behrens, ${ }^{1,2}$ Carlo Pozzilli, ${ }^{3}$ Paul M. Matthews, ${ }^{1,4,5}$ \\ Matthew F. S. Rushworth, ${ }^{1,2}$ and Heidi Johansen-Berg ${ }^{1}$ \\ ${ }^{1}$ Centre for Functional MRI of the Brain and 2Department of Experimental Psychology, University of Oxford, Oxford OX3 9DU, United Kingdom, \\ ${ }^{3}$ Department of Neurological Sciences, "La Sapienza" University, Rome 00185, Italy, ${ }^{4}$ GSK Clinical Imaging Centre, Hammersmith Hospitals, \\ GlaxoSmithKline, London W12 0NN, United Kingdom, and 5Department of Clinical Neurosciences, Imperial College, London W6 8RP, United Kingdom
}

\begin{abstract}
Lateral premotor cortex (PM) in the macaque monkey can be segregated into structurally and functionally distinct subregions, including a major division between dorsal (PMd) and ventral (PMv) parts, which have distinct cytoarchitecture, function, and patterns of connectivity with both frontal and parietal cortical areas. The borders of their subregions are less well defined in the human brain. Here we use diffusion tractography to identify a reproducible border between dorsal and ventral subregions of human precentral gyrus. We derive connectivity fingerprints for the two subregions and demonstrate that each has a distinctive pattern of connectivity with frontal cortex and lateral parietal cortex, suggesting that these areas correspond to human PMd and PMv. Although putative human PMd has a high probability of connection with the superior parietal lobule, dorsal prefrontal cortex, and cingulate cortex, human PMv has a higher probability of connection with the anterior inferior parietal lobule and ventral prefrontal cortex. Finally, we assess the correspondence between our PMd/PMv border and local sulcal and functional anatomy. The location of the border falls at the level of the gyral branch that divides the inferior precentral sulcus from the superior precentral sulcus and corresponded closely to the location of a functional border defined using previous functional magnetic resonance imaging studies.
\end{abstract}

Key words: premotor cortex; diffusion imaging; tractography; human; prefrontal cortex; parietal cortex

\section{Introduction}

In both Old and New World monkeys and prosimian species, the lateral premotor cortex $(\mathrm{PM})$ is separated into distinct functional fields. Two main subdivisions are dorsal PM (PMd) and ventral PM (PMv) (Dum and Strick, 1991; He et al., 1993; Stepniewska et al., 1993; Wu et al., 2000). PMd is critical for learned, nonstandard stimulus-response mappings (Petrides, 1985; Passingham, 1993; Wise et al., 1996), whereas PMv transforms the geometrical properties and spatial location of an object into motor commands (Kurata, 1994; Jeannerod et al., 1995; Hoshi and Tanji, 2006).

The functional specialization is reflected in structural organi-

\footnotetext{
Received May 9, 2007; revised July 17, 2007; accepted Aug. 8, 2007.

This work was supported by the Wellcome Trust (H.J.-B.), the Multiple Sclerosis Society (United Kingdom) (P.M.M., H.J.-B., V.T.), the Multiple Sclerosis Society (Italy) (V.T., C.P.), the United Kingdom Medical Research Council (UK MRC) (T.E.J.B., P.M.M., M.F.S.R.), the Dr. Hadwen Trust for Humane Research (S.J., H.J.-B., T.E.J.B.), the UK Biotechnology and Biological Sciences Research Council (J.C.K.), and the MRC and Royal Society (M.F.S.R.). We acknowledge the generous support of the UK MRC. We thank Matthew Robson for help with sequence programming, Dick Passingham for useful discussions on functional anatomy, and Roberto Toro for help with results rendering.

Correspondence should be addressed to Dr. Heidi Johansen-Berg, Oxford Centre for Functional MRI of the Brain, John Raddliffe Hospital, Headington, 0xford 0X3 0HS, UK. E-mail: heidi@fmrib.ox.ac.uk. D0I:10.1523/JNEUROSCI.2144-07.2007

Copyright $\odot 2007$ Society for Neuroscience 0270-6474/07/2710259-11\$15.00/0
}

zation: PMd and PMv have distinct cytoarchitecture (Matelli et al., 1985; Barbas and Pandya, 1987), receptor distributions (Geyer et al., 1998), and anatomical connectivity. Tracer studies in several nonhuman primate species indicate that they form part of mostly segregated parietal (Matelli et al., 1986, 1998; TanneGariepy et al., 2002; Stepniewska et al., 2006) and prefrontal (Luppino et al., 2003; Dum and Strick, 2005) circuits.

Despite parallels with other primates (Geyer et al., 2000), less is known about structural organization of human PM. Although there have been reports of further divisions (Vogt and Vogt, 1919; Braitenberg, 1962; Zilles et al., 2004), many descriptions of human PM use Brodmann's map (Brodmann, 1909). Neuroimaging of human PM suggests functional divisions previously described in macaque (Toni et al., 2001; Amiez et al., 2006; Beurze et al., 2007), although some have argued for three ventrodorsally organized regions (Schubotz and von Cramon, 2003). There may be a consistent relationship between the location of functional regions and morphology of adjacent sulci such as the precentral sulcus (Germann et al., 2005; Amiez et al., 2006). Whether functional regions in human PM relate to specific parietal and prefrontal anatomical networks, however, is unknown.

Given that connectional anatomy both reflects and determines regional functional organization, patterns of anatomical 
connectivity can inform the segregation of functionally distinct areas (Passingham et al., 2002; Behrens and Johansen-Berg, 2005). Diffusion-weighted magnetic resonance imaging (MRI) and tractography can quantify long-range connectivity in vivo (Catani et al., 2002; Mori and Zhang, 2006; Aron et al., 2007). Tractography techniques subdivided brain structures according to their connectivity patterns in medial frontal cortex [supplementary motor area (SMA)/pre-SMA (Johansen-Berg et al., 2004)], the geniculate bodies [medial vs lateral (Devlin et al., 2006)], and lateral frontal cortex [area 44/area 45/deep frontal operculum (Anwander et al., 2007; Klein et al., 2007)].

Here we used multiple-fiber diffusion tractography (Behrens et al., 2007) to examine the connectivity of human precentral cortex in vivo. A connectivity-defined border separating dorsal and ventral subregions was identified, and its variability and reproducibility were examined. We derived connectivity fingerprints for the two regions and demonstrated that their distinct patterns of parietal and prefrontal connectivity are reminiscent of monkey PMd and PMv. Finally, we examined the relationship between the location of our anatomically defined boundary and both local sulcal landmarks and the functional border reported in previous functional MRI studies (Mayka et al., 2006).

\section{Materials and Methods \\ Data acquisition}

Diffusion-weighted and T1-weighted images were acquired in 17 healthy subjects ( 6 women and 11 men; mean $\pm \mathrm{SD}$ age, $26.18 \pm 4.81$; range, $20-38$ ) on a $1.5 \mathrm{~T}$ Siemens (Erlangen, Germany) Sonata MR scanner, with maximum gradient strength of $40 \mathrm{mT} \cdot \mathrm{m}^{-1}$. All subjects gave informed written consent in accordance with ethical approval from the Oxford Research Ethics Committee.

Diffusion-weighted data were acquired using echo planar imaging $(72 \times 2$-mm-thick axial slices; matrix size, $128 \times 104$; field of view, $256 \times$ $208 \mathrm{~mm}^{2}$; giving a voxel size of $2 \times 2 \times 2 \mathrm{~mm}$ ). Diffusion weighting was isotropically distributed along 60 directions using a $b$ value of 1000 $\mathrm{s} \cdot \mathrm{mm}^{-2}$. For each set of diffusion-weighted data, five volumes with no diffusion weighting were acquired at points throughout the acquisition. Three sets of diffusion-weighted data were acquired for subsequent averaging to improve the signal-to-noise ratio. The total scan time for the diffusion-weighted imaging protocol was $45 \mathrm{~min}$.

A T1-weighted anatomical image was acquired using a threedimensional FLASH (fast low-angle shot) sequence (repetition time, 12 $\mathrm{ms}$; echo time, $5.65 \mathrm{~ms}$; flip angle, $19^{\circ}$; with elliptical sampling of $k$ space, giving a voxel size of $1 \times 1 \times 1 \mathrm{~mm}$ in $5 \mathrm{~min}$ and $5 \mathrm{~s}$ ).

\section{Image analysis}

Analysis was performed using tools from the FMRIB Software Library (FSL) (www.fmrib.ox.ac.uk/fsl) and in-house software.

Preprocessing. We skull-stripped (Smith, 2002) the diffusion-weighted and T1-weighted data and performed affine registration to align diffusion data, T1-weighted scans, and Montreal Neurological Institute (MNI) standard proportional stereotaxic space (Jenkinson and Smith, 2001; Jenkinson et al., 2002). For each subject, T1-weighted scans were processed using FAST (FMRIB's Automated Segmentation Tool) tissuetype segmentation (Zhang et al., 2001) within FSL (www.fmrib.ox.ac.uk/ fsl) to derive partial volume estimates for gray matter (GM) and white matter $(\mathrm{WM})$. These partial volume maps were thresholded at $>0.2$ and dilated by a $1 \times 1 \times 1 \mathrm{~mm}$ voxel. The overlap between the thresholded and dilated GM and WM maps was then used to define a GM/WM boundary region of interest (ROI). Diffusion data were transformed using affine registration to a reference volume to correct for eddy currents and head motion. The data from the three acquisitions were subsequently averaged to improve the signal-to-noise ratio. We calculated probability distributions on multiple fiber directions at each voxel in the diffusion data using a multiple-fiber extension (Behrens et al., 2007) of a previously described diffusion modeling approach (Behrens et al., 2003a,b).
PM ROI. We defined an initial ROI of the PM by hand in each subject's left hemisphere as the cortex and underlying WM in or around the precentral sulcus on the individual T1-weighted images registered to the MNI standard space using FLIRT (Jenkinson and Smith, 2001) within FSL (www.fmrib.ox.ac.uk/fsl). The posterior border was approximately two-thirds of the way across the precentral gyrus (Alkadhi et al., 2002a,b), the inferior border was the ventral limit (Zhang et al., 2001) of the precentral gyrus, and the medial border was $\sim 5 \mathrm{~mm}$ from the medial surface of the brain. The anterior border was located $\sim 5 \mathrm{~mm}$ in front of the precentral sulcus. The decision to place the anterior border of the region considered for parcellation at this position reflected the need for a consistency of the approach and the fact that although functional neuroimaging studies have suggested that human PMd lies posterior to the superior precentral sulcus (Amiez et al., 2006), it has been proposed that a part of human PMv might lie anterior to the inferior precentral sulcus (Geyer et al., 2000). Moreover, in some cases, the dorsal inferior precentral sulcus can be situated anterior to the superior precentral sulcus (Germann et al., 2005). In practice, however, the area that was consistently defined in the initial PM ROI includes the tissue on the precentral gyrus.

To create a starting ROI of equal volume across subjects and hemispheres, manually defined PM ROIs were overlapped across all subjects to create a group overlap PM ROI. This group ROI was mirrored about the midline to create a right-hemisphere PM ROI. The group PM ROIs were then refined for each individual subject by including only those voxels that fell within the GM/WM boundary in that subject. Thus, the final PM ROI for each subject and each hemisphere consisted of the GM/WM boundary within the PM region.

Note that our group PM ROI, defined using sulcal and gyral landmarks, does not overlap precisely with definitions of Brodmann's area 6 (BA6) based on group postmortem data (Geyer, 2004; Eickhoff et al., 2006). For example, the overlap between our ROI and the Juelich Atlas probabilistic map of lateral BA6 (thresholded at 10\%) is 66\% in the left hemisphere and $50 \%$ on the right hemisphere. This is a consequence of the particular care we took to exclude regions from the premotor mask that could fall within primary motor cortex. Note that there is considerable overlap between the probabilistic locations of the Juelich premotor and primary motor areas because of interindividual anatomical variability. Our ROI is shifted anteriorly with respect to the Juelich Atlas definition of BA6 because it avoids overlap with the primary motor cortex.

Probabilistic multifiber diffusion tractography. For each subject, probabilistic tractography was run from voxels at the GM/WM boundary within the PM seed area to assess connectivity with every brain voxel, using a model accounting for multiple fiber orientations in each voxel (Behrens et al., 2007). The approach draws a sample from each fiber orientation distribution at the current voxel and chooses the sample closest to the orientation of its previous step. The connection probability between a PM voxel and another voxel in the brain is defined as the sum of sample fiber lengths connecting these two voxels. This value corresponds approximately to the connection probability multiplied by the average connection length. It corrects for the fact that distant regions appear less connected than close regions when using the probability alone and is appropriate for parcellation based-studies in which distant connections are the principle focus, and quantitative probability values are not required. For each subject, we drew 1000 samples from the connectivity distribution from each seed voxel. The algorithm was limited to estimating two fiber orientations at each voxel, because of the $b$ value and number of gradient orientations in the diffusion data (Behrens et al., 2007).

Connectivity-based parcellation. A connectivity matrix, A, between PM seed voxels and each other brain voxel was derived as described previously (Johansen-Berg et al., 2004; Klein et al., 2007) and used to generate a symmetric cross-correlation matrix, $\mathbf{B}=\mathbf{A A}^{\mathbf{T}}$, of dimensions (number of seeds $\times$ number of seeds) in which the $(i, j)$ th element value is the correlation between the connectivity profile of seed $i$ and the connectivity profile of seed $j$. The rows in $\mathbf{B}$ were permuted using $k$-means segmentation for automated clustering to define two regions (Anwander et al., 2007; Klein et al., 2007). The goal of clustering the cross-correlation matrix $\mathbf{B}$ is to group together regions that share the same connectivity with the rest of the brain. However, it is possible for two regions to be 
grouped together because they are strongly connected to each other (even if their connections to other cortical regions are very different). The connection they share is a connection between them, rather than connections with the rest of the brain. To correct for that, we included a distance constraint, by adding a (scaled) Euclidean distance matrix to the cross-correlation matrix. The resulting clusters are then constrained to consist of voxels that are spatially contiguous, although the border between clusters is still guided by remote connectivity information.

The automated classification using $k$-means clustering produced individual parcellation of the left and right PM into dorsal and ventral subregions (PMd and PMv, respectively) as groups of elements that were strongly correlated with each other and weakly correlated with the rest of the matrix. Results from clustering are mapped back onto the brain. The two PM subregions were used as masks for assessing variability in PM parcellation and defining connectional fingerprints.

Intersubject variability and intersession reproducibility. A subcohort of the subjects (eight subjects, four women; age range, 21-34 years) underwent a reproducibility study. Each of them was scanned three times on different days, using the same scanner and settings. The general issue of between-session reproducibility and between-subject variability of diffusion MR, tractography, and parcellation measures has been addressed previously in the same cohort of subjects (Heiervang et al., 2006; Klein et al., 2007). In the present study, we test specifically for the variability of the PM parcellation and the location of the boundaries between its subregions in each hemisphere. First, we tested for agreement of segmented volumes between sessions by calculating the conditional probability that a voxel classified as belonging to a particular region in session A would receive the same classification in session $B$. This was calculated separately for PMd and PMv as $P_{\mathrm{A}}(B)=$ (number of overlapping voxels between segmentation for session $\mathrm{A}$ and session $\mathrm{B}$ )/(number of voxels in segmentation for session $B)$. For each subject and each pair of acquisitions, we then calculated the average of $P_{\mathrm{A}}(B)$ and $P_{\mathrm{B}}(A)$. Second, we tested for reproducibility of boundary location by automatically computing a plane dividing the PMd and the PMv using a linear support vector machine (SVM). We used SVM-light software (Joachim, 1999) to compute the support vectors from the data. Group results of the connectivitydefined PMd/PMv boundary were mapped to (three-dimensional) surface reconstruction using one of the individual structural images as a template, which is registered to the MNI standard space (Brett et al., 2002).

Prefronto-parietal connectional fingerprints. To further investigate the differences in the anatomical networks involving PMv and PMd, and to compare them to results from animal models, we evaluated the connectivity patterns from PMd and PMv to ipsilateral parietal and prefrontal cortical areas. We used hand-drawn masks of subregions of left prefrontal and parietal cortical areas, the connectivity profiles of which were originally described previously in a subgroup of our population (Croxson et al., 2005; Rushworth et al., 2006) (see Fig. 3). Prefrontal ROIs included the following: ventrolateral prefrontal cortex (PFvl), dorsolateral prefrontal cortex (PFdl), dorsomedial prefrontal cortex (PFdm), cingulate sulcus, cingulate gyrus, medial orbital cortex, central orbital cortex, and lateral orbital cortex. Parietal ROIs included the following: medial intraparietal sulcus (IPS), anterior inferior parietal lobule and adjacent anterior intraparietal sulcus (AIPL), angular gyrus (ANG), superior parietal lobule (SPL), medial bank of the descending IPS, and lateral bank of the descending IPS. The AIPL mask was centered on the AIPL-IP2 mask from the Juelich cytoarchitectonic probabilistic atlas (Choi et al., 2006). Previously defined population maps of manually defined ROIs were thresholded to include only those voxels present in $>50 \%$ of the population for each ROI and then dilated by a $1 \times 1 \times 1$ $\mathrm{mm}$ voxel. To refine these ROIs for each of the subjects in the current study, we masked the population ROIs by individually defined GM/WM boundaries. To create right-hemisphere ROIs, the left dilated population masks were mirrored about the midline and masked by individualsubject GM/WM boundaries. For each subject, we drew 5000 samples from the connectivity distribution from each seed voxel (connectivitydefined PMv or PMd) and computed the probability of connection to each cortical target mask (parietal and prefrontal ROIs). For each subject, the mean probability of connection between the seed region (connectivity-defined PMv or PMd) and each target region (parietal and prefrontal ROIs) was computed. We then normalized these values by dividing by the total sum of probability to any target mask and multiplying by 100 so that these values represent connection probability as a percentage of total connections (Croxson et al., 2005). Any target mask with a normalized connectivity probability that was $<0.2 \%$ of the sum of all target probabilities for both premotor seed regions was excluded from additional analyses. Connection probabilities of those regions, which survived thresholding, were divided by the size of the target ROIs, computed on an individual-subject basis, to produce connection probabilities adjusted for the size of the seed regions as well as for the volume of the targets. These normalized values were then used to generate connectivity fingerprints.

\section{Statistical analysis}

Statistical analysis on connection probability was performed using SPSS 11 (SPSS, Chicago, IL). Repeated-measures ANOVAs of connection probability values were used to test for the influence of within-subject factors of hemispheres (left vs right), PM subregions (PMd vs PMv), and target ROI (nine prefronto-parietal ROIs). Paired $t$ tests were used to compare volumes of connectivity-defined PM subregions and to follow-up on ANOVAs of connection probabilities.

\section{Results}

\section{Connectivity-based parcellation of the PM}

Parcellation of left and right PM on the basis of sharp changes in the connectivity profiles identified two clusters in the reordered connectivity cross-correlation matrices, which represent a group of seed voxels with a similar pattern of connectivity (Fig. 1). When mapped back onto the brain, the voxels in the two clusters corresponded to discrete, spatially contiguous subregions along the lateral surface of PM (Fig. 1). The boundary between PMd and PMv was located at an average of $z$ coordinate of 48 in both the left (range in individual subjects, $z=42$ to $z=56$ ) and the right (range, $z=40$ to $z=56$ ) hemisphere.

We compared the volumes of connectivity-defined dorsal and ventral subregions in each hemisphere. The volume of the PM ROI was greater in the right than the left hemisphere (1409.18 \pm 137.30 vs $1316.24 \pm 197.22$ mean \pm SD number of voxels; $t=$ 3.248; $p<0.005$ ). Note that because the starting volume was identical for all subjects and for both hemispheres, this size asymmetry must reflect hemisphere differences in the relative size of the GM/WM boundary (an index of apparent neocortical thickness). After parcellation of the PM ROI into dorsal and ventral subregions, repeated-measures ANOVA found a main effect of hemisphere $(F=10.6$; $\mathrm{df}=1.00,16.00 ; p<0.05)$ and PM subregion $(F=34.8 ; \mathrm{df}=1.00,16.00 ; p<0.001)$ and a trend toward an interaction between the two $(F=3.6 ; \mathrm{df}=1.00,16.00 ; p=0.086)$. The main effect of PM subregion reflected the finding that the dorsal region was significantly larger than the ventral region in both hemispheres (left: $749.41 \pm 79.80$ vs $566.65 \pm 131.16$ voxels, $t=8.3, p<0.001$; right: $763.18 \pm 90.51$ vs $646.00 \pm 116.35$ voxels, $t=3.1, p<0.01)$. The trend toward an interaction between hemisphere and subregion reflected the finding that the ventral region was significantly larger in the right hemisphere $(646.00 \pm 116.35)$ compared with the left hemisphere $(566.65 \pm$ $131.16 ; t=3.7 ; p<0.005)$, whereas no significant difference was found between the volumes of the dorsal subregions in the left $(749.41 \pm 79.80)$ and $\operatorname{right}(763.18 \pm 90.51 ; t=-0.6 ; p=\mathrm{NS})$ hemispheres.

To assess the reproducibility of the PM parcellation, eight subjects were scanned on 3 separate days. Average intersession reproducibility of the segmented volumes across scanning days was $98.6 \%( \pm 1.1)$ for the dorsal subregion and $99.1 \%( \pm 2.1)$ for the ventral subregion (Fig. 2a). Similarly, the angles between 
Sbj 1
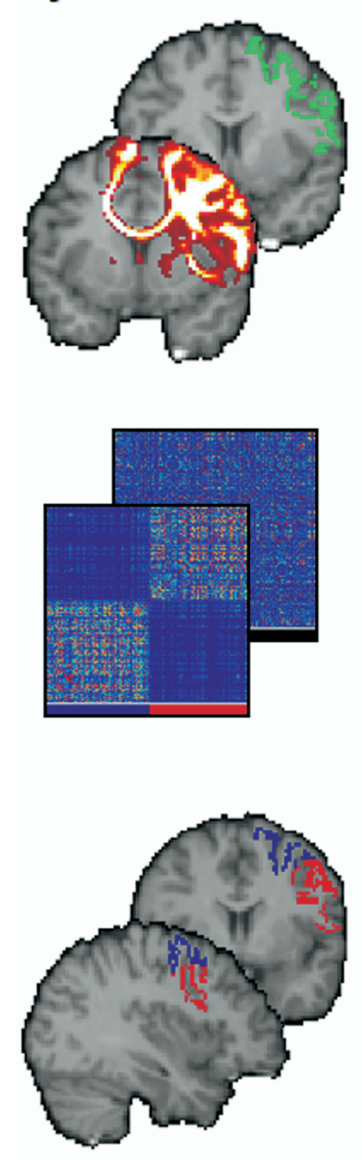

a.

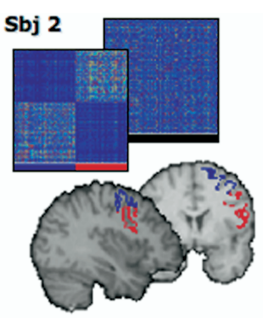

Sbj 6

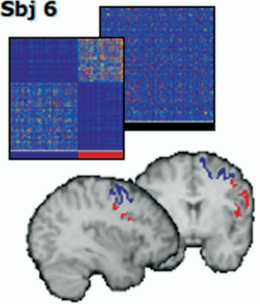

Sbj 10

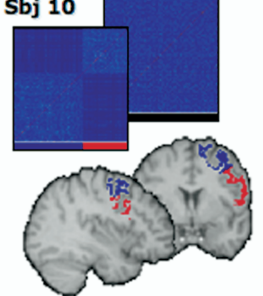

Sbj 14

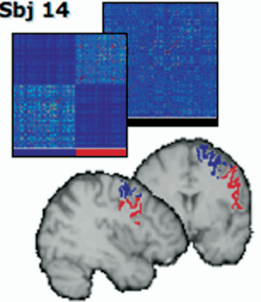

Sbj 3

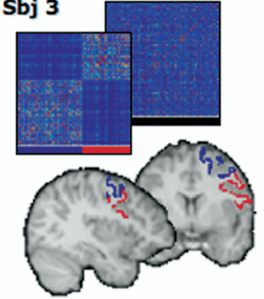

Sbj 7
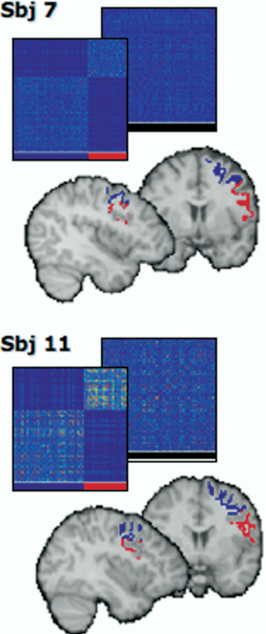

Sbj 15

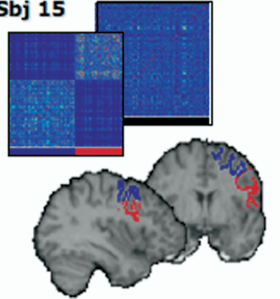

Sbj 4

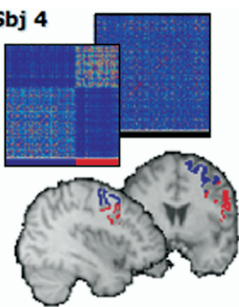

Sbj 8
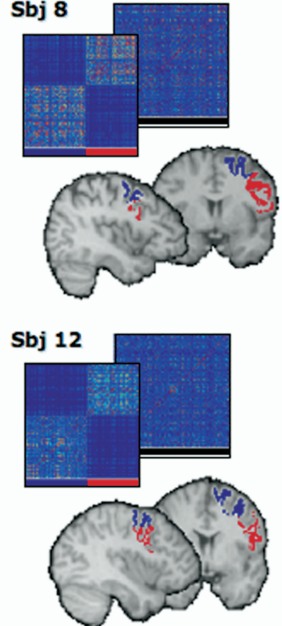

Sbj 16

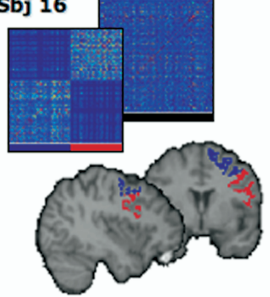

Sbj 5

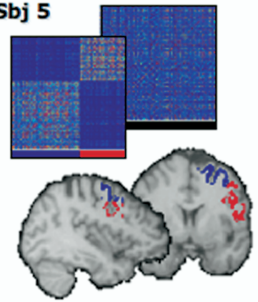

Sbj 9
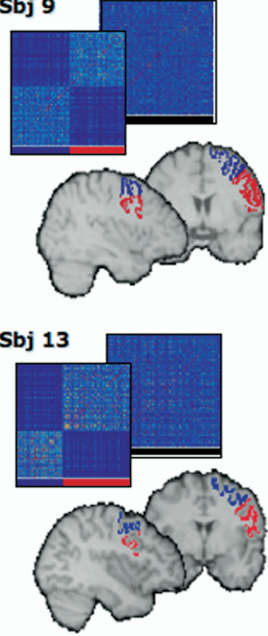

Sbj 17

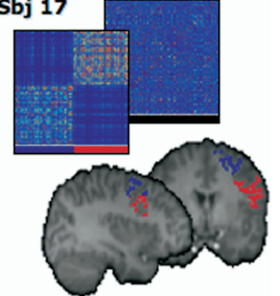

Figure 1. $\quad \boldsymbol{a}$, Summary of the steps leading to the PMd/PMv clustering in an example subject (Sbj). Top, PM region (green) and the resulting tracts. Middle, Cross-correlation matrix (and its reorganized counterpart) indicating the degree of similarity in connectivity pattern between the voxels in PMd and PMv, respectively. Bottom, The two resulting clusters on a coronal ( $y=63$ ) and a sagittal $(x=63)$ slice. $\boldsymbol{b}$, Results of the connectivity-based clustering for the other 16 subjects. Note the recurrent presence of a sharp border between the two clusters.

planes identifying PMd/PMv boundaries were consistent within a subject, as well as across subjects, varying much less within a subject than across subjects (Fig. $2 b$ ).

\section{Parieto-frontal connectivity fingerprints of PM subregions}

If the subregions we identified correspond to PMd and PMv, then tracer studies on PM connections in nonhuman primates provide strong previous hypotheses regarding expected differences in parieto-frontal connectivity patterns for the two regions (Rizzolatti et al., 1998). We therefore quantified the probabilities of connection from our connectivity-defined dorsal and ventral subregions to a number of parietal and prefrontal ROIs to test for these anatomical relationships in the human brain (Fig. 3). Of the 13 target ROIs tested, 9 had a $>0.2 \%$ probability of connection to both of the premotor seed regions (Table 1). The connection probabilities for these nine regions were normalized for the size of individual target ROIs (Table 2). Normalized connection probabilities were entered into a repeated-measures ANOVA that revealed a main effect of $\mathrm{PM}$ subregions $(F=46.6$; $\mathrm{df}=$ $1.00,16.00 ; p<0.001)$ and target ROI $(F=245.9 ; \mathrm{df}=$ 2.26,36.20; $p<0.001)$ and a significant interaction between PM subregions and target ROI $(F=89.7 ; \mathrm{df}=2.98,47.67 ; p<0.001)$, a reflection of the difference in the patterns of parieto-frontal connectivity between the dorsal and ventral subregions (Fig. 3).
Significant interactions with hemisphere were also found and are discussed below.

Our anatomically defined dorsal region had particularly high probabilities of connection with cingulate sulcus, cingulate gyrus, SPL, and the IPS, whereas the ventral subregion showed particularly high probabilities of connection with the ventrolateral prefrontal cortex, anterior inferior parietal lobe (supramarginal gyrus), as well as connectivity with the posterior ANG and lateral parts of the orbital prefrontal cortex. Direct comparison of connectivity probabilities between dorsal and ventral subregions revealed higher probabilities of connection for dorsal compared with ventral in both hemispheres for the cingulate gyrus (left: $t=$ $4.3, p<0.001$; right: $t=3.2, p<0.01$ ), cingulate sulcus (left: $t=$ $3.4, p<0.005$; right: $t=4.5, p<0.001$ ), and SPL (left: $t=3.1, p<$ 0.01 ; right: $t=3.3, p<0.01)$ and in the left hemisphere only for dorsolateral prefrontal cortex (left: $t=2.9, p<0.05)$ and IPS (left: $t=3.3, p<0.01$ ). The ventral region had significantly higher connection probability than the dorsal region in both hemispheres for ventrolateral prefrontal cortex (left: $t=13.4$, $p<0.001$; right: $t=14.2, p<0.001)$, anterior intraparietal lobule (left: $t=3.7, p<0.01$; right: $t=4.2, p<0.01$ ), lateral orbitofrontal cortex (left: $t=2.9, p<0.05$; right: $t=4.2, p<0.01$ ), and in the right hemisphere only for ANG (right: $t=3.2, p<0.01$ ).

Repeated-measures ANOVA of connection probabilities also 


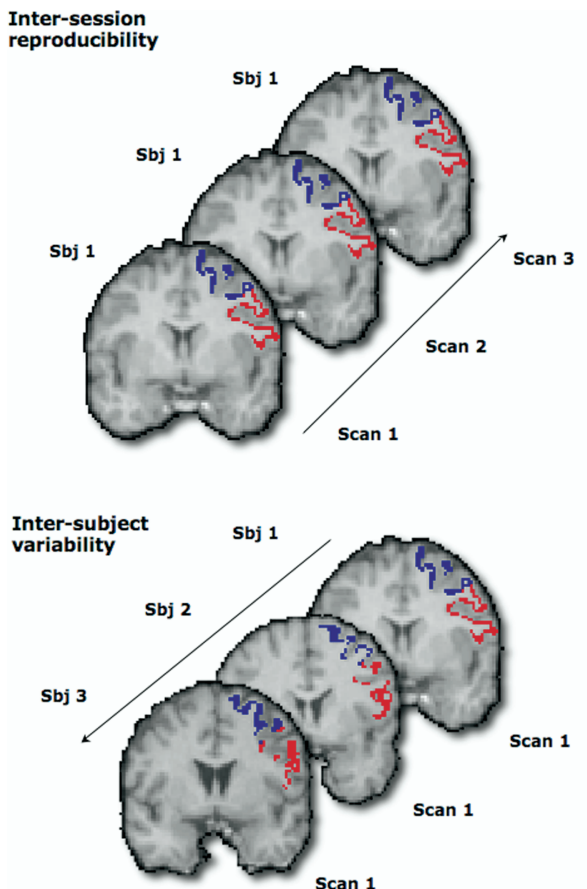

a.

Figure 2. Results of the reproducibility study. $\boldsymbol{a}$, Schematic representation of the intersession reproducibility (same subject, different scanning sessions) and the intersubject variability (different subjects, same session). Sbj, Subject. $\boldsymbol{b}$, Top, Planes dividing PMd and PMv automatically calculated by the SVM classifier for subject 1 (red; three sessions) and subjects 2 - 8 for session 1 (blue) on the left hemisphere. Bottom, For each subject, the ordinate represents the angle in degrees between the plane identified from session 1 and the other sessions (red; intrasubject variability) or the other subjects for all sessions (blue; intersubject variability). The figure illustrates that there is little variability between subjects and even less variability within subjects. $p<0.05)$. Note that the connection probability values are normalized for both seed and target volume, so that these hemispheric asymmetries in the connection probabilities between seed regions and target ROIs exist over and above differences in mask volumes reported previously.

One potential confound for hemispheric asymmetries in connection probabilities would be ease of tracking parietal-frontal pathways past the corticospinal tract. To test whether this could be influencing our results, we defined two pairs of ROIs in each hemisphere within the second and third branches of the superior longitudinal fasciculus, which connect parietal and frontal cortical areas. By positioning ROIs within each tract $\sim 2 \mathrm{~cm}$ anterior and posterior to the corticospinal tract, we were able to test ease of tracking within the tract in each hemisphere. We did not find any significant hemispheric asymmetries in tracking performance.

\section{Comparison with sulcal and functional anatomy}

To compare the location of our connectivity-defined border to local sulcal anatomy, a plane dividing the dorsal and the ventral subregions was automatically computed in each subject and projected onto individual, as well as onto group, cor-

found a significant two-way interaction between hemisphere and parieto-frontal target ROIs $(F=4.17$; $\mathrm{df}=3.77,60.28$; $p<$ $0.001)$, as well as a three-way interaction between hemisphere, PM subregions, and target ROIs $(F=3.46$; $\mathrm{df}=2.66,42.55 ; p<$ 0.05). Follow-up ANOVAs considering each PM subregion separately showed that although the ventral region connection probabilities (pooled across all target ROIs) were roughly symmetrical (left ventral, $0.367 \pm 0.014$ vs right ventral, $0.366 \pm 0.014$ ), connection probabilities for the dorsal subregion showed a trend to be higher in the left hemisphere (left dorsal, $0.282 \pm 0.017 \mathrm{vs}$ right dorsal, $0.254 \pm 0.018 ; F=3.63$; df $=1.00,16.00 ; p<0.1$. Follow-up ANOVAs considering each target ROI separately showed that although most targets did not show any hemispheric asymmetry in connection probability, others were significantly lateralized. Specifically, we found a greater connection probability in the left hemisphere than the right for PFvl $(F=4.26$; $\mathrm{df}=$ $1.00,16.00 ; p=0.056)$ and PFdl $(F=10.39 ; \mathrm{df}=1.00,16.00 ; p<$ $0.01)$, whereas we found greater connection probabilities in the right hemisphere for the ANG $(F=5.21$; df $=1.00,16.00 ; p<$ $0.05)$ and IPS $(F=8.05 ; \mathrm{df}=1.00,16.00 ; p<0.05)$. Some of these hemispheric asymmetries varied between PM subregions: for PFdl, we also found an interaction between hemisphere and PM subregion $(F=5.51$; $\mathrm{df}=1.00,16.00 ; p<0.05)$, reflecting the fact that the left lateralization of PFdl connection probabilities was significant for the dorsal subregion $(t=4.7 ; p<0.001)$ but not for the ventral region $(t=0.07$; NS). For ANG, we also found an interaction between hemisphere and subregion $(F=9.15$; $\mathrm{df}=$ $1.00,16.00 ; p<0.01$ ), now reflecting the finding that, although connection probabilities of the dorsal region were roughly symmetrical ( $t=-0.62$; NS), connection probabilities of the ventral region were greater in the right hemisphere than the left $(t=2.92$; tical surfaces (Fig. 4). In both hemispheres, the projection of this boundary tended to lie dorsal to the horizontal extension of the inferior precentral sulcus and adjacent to the most ventral part of the superior precentral sulcus, locating the boundary between the levels of the inferior and superior frontal sulci, consistent with the hypothesized location of the PMd/PMv border in the human brain.

To compare our connectivity-defined border to local functional anatomy, we overlaid our results onto data from a metaanalysis of functional studies (Mayka et al., 2006), which reconstructed spatial probability maps based on the location of the activations in regions designated as PMd and PMv in $>100$ different functional imaging studies. The functional border between PMd and PMv colocalized extremely well with our connectivitybased border (Fig. 5), confirming the hypothesis that subregions identified by differences in their connectivity profiles have different functional specializations and that they correspond to physiologically defined PMd and PMv. Some of the strongest evidence for functional localization in PM comes from studies of standard and nonstandard stimulus response mappings, found to activate PMv and PMd, respectively. To test whether our connectivitybased mapping is consistent with these established roles for PMd and PMv, we compared locations of functional activation from three studies of conditional motor selection (Toni et al., 2001; Amiez et al., 2006; Grol et al., 2006) and three studies of reaching and grasping (Binkofski et al., 1999b; de Jong et al., 2001; Ehrsson et al., 2003). When projecting these activations onto our connectivity-based map at $y=0$, activations for conditional motor selection (i.e., nonstandard mappings) fell within putative PMd, whereas those for reaching and grasping (i.e., standard mappings) fell within putative PMv. 
$\square$ dorsal $\square$ ventral

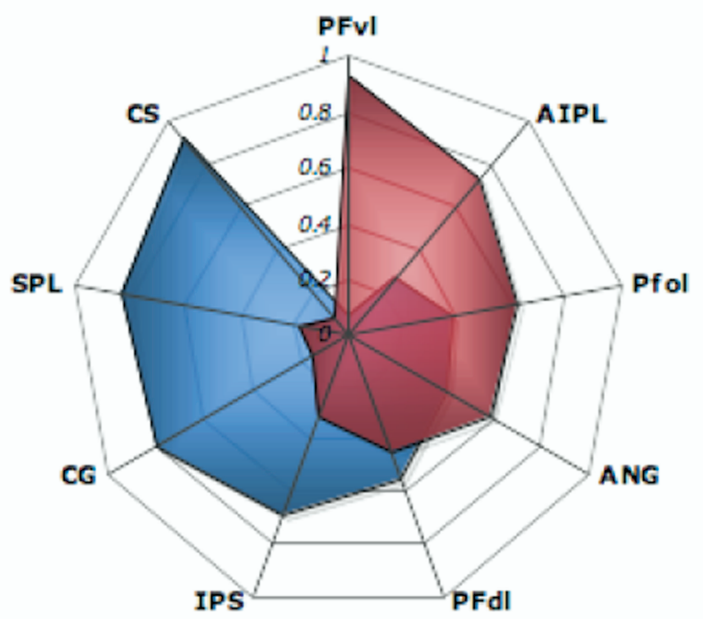

\section{RIGHT HEMISPHERE}

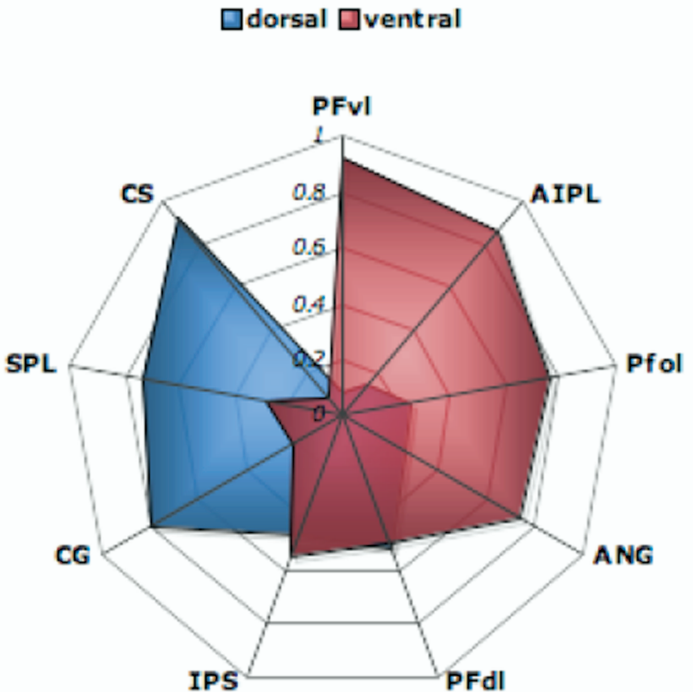

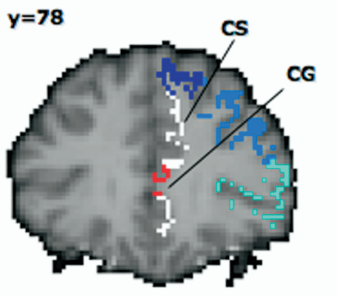
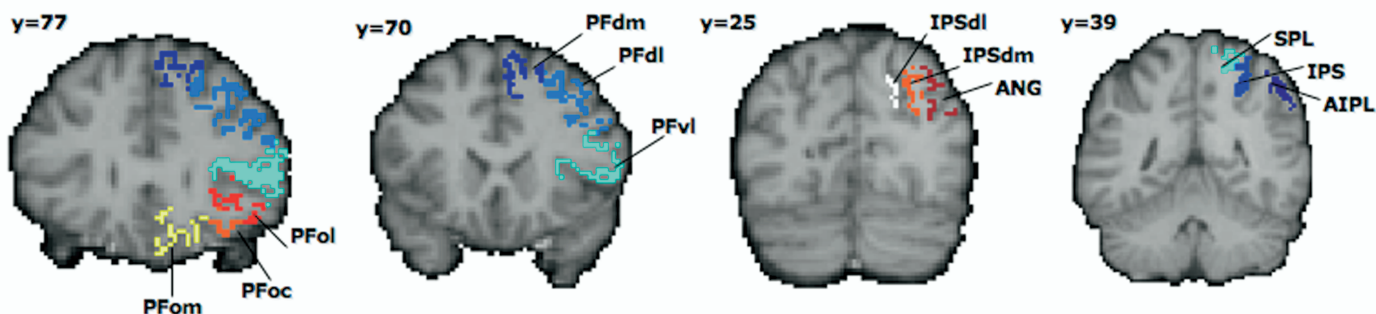

Figure 3. Connectivity fingerprints in the left hemisphere (top left) and the right hemisphere (top right). The values indicate the relative connection probability between PMd (blue) or PMv (red) and the prefronto-parietal targets, the masks of which are shown in the bottom row. Abbreviations as in Table 1.

Table 1. Average (across subjects) connection strength between each seed region (left and right PMv or PMd) and each target region (parietal and prefrontal ROIs)

\begin{tabular}{|c|c|c|c|c|c|c|c|c|c|c|c|c|c|}
\hline & CG & $C S$ & PFom & PFoc & PFol & PFvl & PFdl & AIPL & ANG & IPS & IPS_dl & IPS_dm & $\overline{S P L}$ \\
\hline IPMd & 1.94 & 2.77 & 0.04 & 0.04 & 0.08 & 2.07 & 40.39 & 0.31 & 0.35 & 0.51 & 0.12 & 0.09 & 0.50 \\
\hline IPMv & 0.26 & 0.09 & 0.05 & 0.11 & 0.24 & 42.19 & 50.37 & 1.57 & 0.94 & 0.40 & 0.05 & 0.04 & 0.15 \\
\hline rPMd & 1.56 & 2.83 & 0.04 & 0.05 & 0.08 & 2.30 & 39.28 & 0.19 & 0.39 & 0.70 & 0.05 & 0.06 & 0.30 \\
\hline rPMv & 0.20 & 0.06 & 0.03 & 0.12 & 0.38 & 38.61 & 51.61 & 1.82 & 1.48 & 1.07 & 0.06 & 0.04 & 0.17 \\
\hline
\end{tabular}

Data are percentages. Each value is divided by the sum of connections to all the targets, so as to be expressed as a percentage of its contribution to the total connections to all the targets. Any target mask with a contribution that was $<0.2 \%$ for both PMv and PMd was excluded from further analyses. Note that the target region volumes are different for left and right seeds. IPMd, Left PMd; IPMv, left PMv; rPMd, right PMd; rPMv, right PMv; CG, cingulate gyrus; CS, cingulate sulcus; PFom, medial orbital prefrontal cortex (PF); PFoc, central orbital PF; PFol, lateral orbital PF; PFvl, ventrolateral PF; IPS_dl, lateral bank of the descending IPS; IPS_dm, medial bank of the descending IPS.

Table 2. Average (across subjects) normalized connection strength between each seed region (left and right PMv or PMd) and each target region (parietal and prefrontal ROIs)

\begin{tabular}{llllllllll}
\hline & CG & CS & PFol & PFvl & PFdl & AIPL & ANG & IPS & SPL \\
\hline IPMd & 0.44 & 0.18 & 0.02 & 0.12 & 1.47 & 0.06 & 0.07 & 0.08 & 0.16 \\
IPMv & 0.04 & 0.00 & 0.03 & 1.73 & 1.16 & 0.20 & 0.12 & 0.04 & 0.03 \\
rPMd & 0.41 & 0.20 & 0.02 & 0.12 & 1.25 & 0.03 & 0.08 & 0.12 & 0.09 \\
rPMv & 0.03 & 0.00 & 0.05 & 1.49 & 1.15 & 0.21 & 0.23 & 0.13 & 0.03 \\
\hline
\end{tabular}

Connection probabilities have been normalized dividing by individual target ROI volumes. Abbreviations are as for Table 1.

\section{Discussion}

Our results allow us to draw three main conclusions. First, we can differentiate reproducible dorsal and ventral subregions of human PM on the basis of their anatomical connection patterns. Second, as in macaque monkey, the two human subregions have distinct connections with parietal and prefrontal areas, suggesting that they correspond to functional areas PMd and PMv. Fi- nally, the boundary between the two subregions based on anatomical connectivity lies in a consistent location with respect to local sulci and to local functionally-defined areas, providing additional support that our regions correspond to PMd and PMv. Our finding that tractography defines reproducible and functionally relevant subregions and borders in PM supports the generality of an approach previously used in other brain regions 


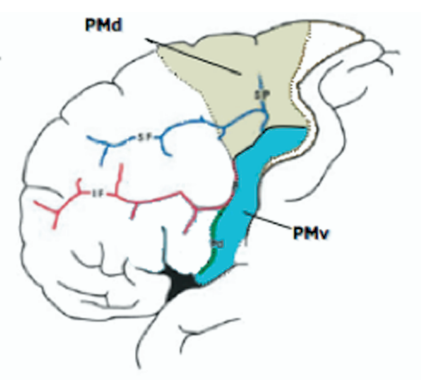

Sbj 1

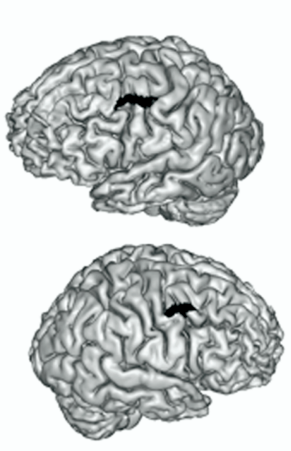

Sbj 5
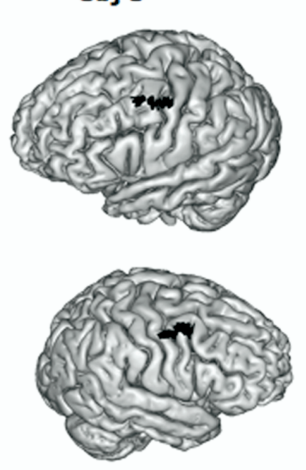

Sbj 2
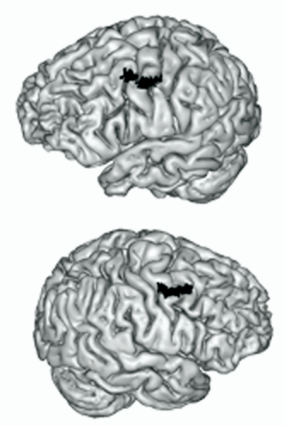

Sbj 3

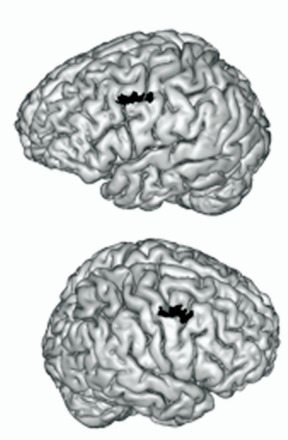

Sbj 4

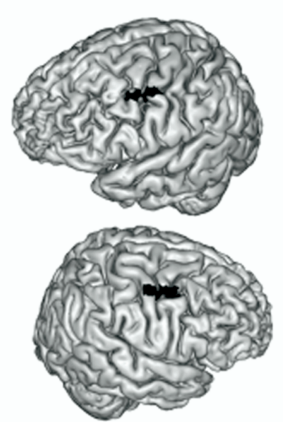

Sbj 7

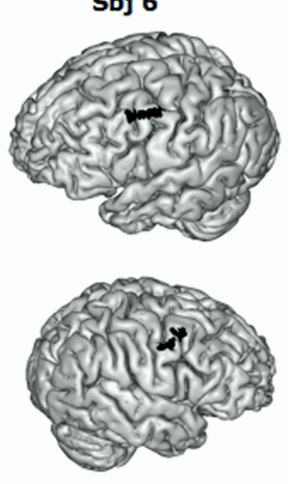

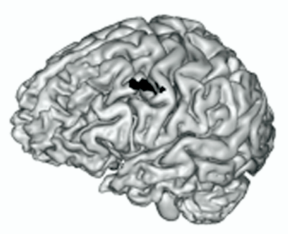

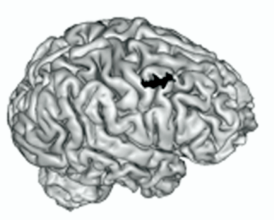

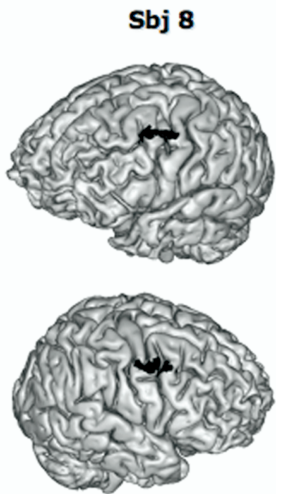

a.

b.

Figure 4. a, Top, Hypothesized subdivision of human PM (modified from Geyer et al., 2000). Bottom, Group map of the border between PMd and PMv displayed on the three-dimensional cortical surface from one individual structural image. The color scale indicates the number of subjects sharing a given location (top, lefthemisphere; bottom, right hemisphere).SP, Superior precentral sulcus; IPd, dorsal part of the inferior precentral sulcus; IPv, ventral part of the inferior precentral sulcus; IF, inferior frontal sulcus; $S F$, superior frontal sulcus; HE, horizontal extension of inferior frontal sulcus. $\boldsymbol{b}$, Overlay of the left and right PMd/PMv borders on the individual three-dimensional cortical surfaces. In the majority of cases, the border lies between the superior and the inferior parts of the precentral sulcus. Sbj, Subject.

(Johansen-Berg et al., 2004; Anwander et al., 2007; Klein et al., 2007).

The parcellation procedure provides a measure of the distinctiveness of connections of the areas, but it does not reveal which connections are critical determinants of the difference. Based on tracer studies on PMd and PMv in nonhuman primates (Matelli et al., 1986, 1998; Tanne-Gariepy et al., 2002; Luppino et al., 2003; Dum and Strick, 2005), we hypothesized that connectivity differences would reflect variation in connections with parietal and frontal brain regions. Probabilistic tractography was therefore used to assess connectivity for all voxels within each subject's PM subregions and a set of masks dividing the prefrontal and parietal cortical regions into component regions investigated in previous studies (Croxson et al., 2005; Rushworth et al., 2006).

Within parietal cortex, human PMd was most strongly connected with the superior parietal lobule and adjacent IPS (Fig. 5, SPL), in line with previous studies in Old and New World monkeys and prosimians reporting strong PMd inputs from superior parietal areas, particularly medial intraparietal sulcal divisions MIP and PEip, and posterior SPL, medial SPL, and parietooccipital areas PEc, PGm, and V6A (Tanne et al., 1995; Johnson et al., 1996; Wise et al., 1997; Matelli et al., 1998; Fang et al., 2005;
Stepniewska et al., 2006). In contrast, our results suggest human $\mathrm{PMv}$ is mainly interconnected with the inferior parietal lobule (IPL). Although there were some connections with the ANG, many were with its more anterior parts, and the highest connection probability was with the AIPL mask, which included the supramarginal gyrus and adjacent anterior lateral IPS. This is consistent with reports of strong inputs to macaque PMv from anterior intraparietal region AIP and the anterior IPL convexity areas PF and PFG (Matelli et al., 1986; Cavada and GoldmanRakic, 1989; Luppino et al., 1999; Lewis and Van Essen, 2000; Rozzi et al., 2006). There was also evidence for more limited PMv and PMd connections with a more posterior IPS region (Fig. 5, IPS). This region has high connection probability with superior colliculus and functional similarities with macaque LIP (Silver et al., 2005; Rushworth et al., 2006). There is evidence for limited connections between LIP and both premotor areas in macaque (Luppino et al., 1999; Lewis and Van Essen, 2000).

As for prefrontal connections, human PMd had a high probability of connection to dorsal prefrontal areas, but there was little evidence of connectivity with ventral and orbital prefrontal cortex. The findings are consistent with reports that macaque rostral $\mathrm{PMd}$, including area F7, is strongly connected with dorsolateral 


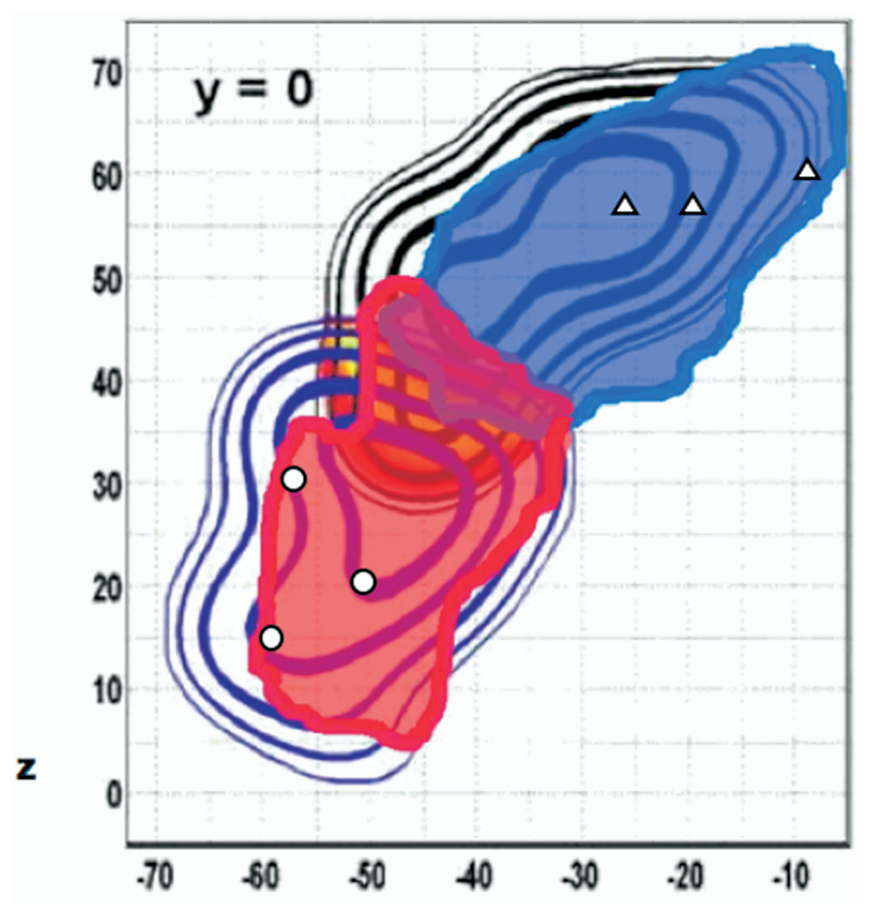

$\mathbf{x}$

Figure 5. Overlay of the group PMd/PMv parcellation (foreground) on the functional MR mapping given by the meta-analysis of Mayka et al. (2006) (background). It shows a very close correspondence between the functional and the connectivity-based borders of the two premotor subregions. Open symbols represent the $x, z$ coordinates of functional activations from specific individual studies projected onto our connectivity-based map at $y=0$. Triangles represent activations from three studies of conditional motor selection (Toni et al., 2001; Amiez et al., 2006; Grol et al., 2006) that fall within putative PMd (blue), and open circles represent locations of functional activations from three studies of reaching and grasping (Binkofski et al., 1999b; de Jong et al., 2001; Ehrsson et al., 2003) that fall within putative PMv (red).

prefrontal cortex but not ventrolateral and orbitofrontal cortex (Barbas and Pandya, 1987; Lu et al., 1994; Takada et al., 2004). Human PMd was also found to be connected to the cingulate sulcus and adjacent gyrus, and again similar connections have been reported in the monkey (Van Hoesen et al., 1993; Hatanaka et al., 2003; Luppino et al., 2003; Takada et al., 2004; Dum and Strick, 2005). In contrast, we found that the most probable connections of human PMv were with ventrolateral and orbital prefrontal areas. In Old and New World monkeys, both anterior $\mathrm{PMv}$ and posterior PMv are connected with prefrontal cortex. There is little evidence for connections with dorsal prefrontal cortex, but the ventral part of the sulcus principalis and the ventral convexity are connected with $\mathrm{PMv}$, as is a region including frontal opercular cortex and gustatory insula that extends toward the lateral orbital surface (Barbas and Pandya, 1987; Preuss and Goldman-Rakic, 1989; Carmichael and Price, 1995; Cipolloni and Pandya, 1999; Takada et al., 2004; Dum and Strick, 2005). Not only were the recorded connection probabilities consistent with what is known of connections in other primates, but absences of connections were also notable. For example, as in macaque, we did not find evidence of significant connectivity between either premotor region and medial orbital prefrontal areas.

In summary, despite some overlap in PMd and PMv connection probabilities with prefrontal and parietal regions, our results confirm that each PM subregion participates in distinct prefronto-parietal circuits, providing an anatomical substrate for their functional specializations. A connection between PMv and human AIP could underlie the involvement of both regions in reaching, grasping, manual exploration, and the representation of body position and ownership (Binkofski et al., 1999a; Grefkes et al., 2002; Ehrsson et al., 2005). Macaque AIP and PMv have been associated with similar functional roles (Gallese et al., 1994; Graziano, 1999; Fogassi et al., 2001). AIP may be one source of visual information used to guide movements (Nakamura et al., 2001). Connections between SPL and medial IPS areas and PMd are thought to be important during reaching (Caminiti et al., 1996; Pesaran et al., 2006) and learning arbitrary stimulus-response rules (Wise et al., 1997). Similarly, human SPL is active when subjects reach and when conditional action selection rules are used (Astafiev et al., 2003; Grol et al., 2006). Connection patterns with frontal regions are also likely to determine the functional roles of PM areas (e.g., ventral prefrontal and cingulate regions in the macaque exert distinct influences over action selection) (Rushworth et al., 2005; Kennerley et al., 2006).

We tested the functional significance of our connectivitydefined boundary by comparing our results with independent spatial probability maps of regions designated as PMd and PMv in functional imaging studies (Mayka et al., 2006). The functionally defined border colocalized with our connectivity-defined boundaries (Fig. 5), confirming the general hypothesis that subregions identified by differences in connectivity profiles have different functional specializations (Passingham et al., 2002; Johansen-Berg et al., 2004, 2005; Friederici et al., 2006).

By projecting the $\mathrm{PMd} / \mathrm{PMv}$ boundary onto individual brain surfaces, we tested the correspondence between connectional anatomy and local sulci. This is an issue of general interest in neuroanatomy (Amiez et al., 2006). Indeed, some hypotheses propose that sulcal folding is a direct consequence of connectional architecture (Van Essen, 1997). In most subjects, the PMv/ PMd border was located dorsal to the horizontal extension of the inferior precentral sulcus and adjacent to the most ventral part of the superior precentral sulcus, corresponding to a mean location of $z=48$, supporting predictions from macaque (Rizzolatti et al., 1998) and human (Rizzolatti et al., 2002) studies.

There was evidence for differing patterns of hemispheric asymmetry in PMv and PMd, because the PMv volume was larger in the right hemisphere. Watkins et al. (2001) reported higher GM density in a right ventral frontal region, including the PMv region defined here. They argued that this was one of the regions underlying the right frontal petalia. The right inferior frontal region that is particularly active when movements are inhibited is centered on PMv and the pars opercularis region immediately anterior (Aron and Poldrack, 2006).

When testing for asymmetries in connection patterns (after normalizing for volumetric differences), we found that although PMd showed a trend for higher connection probabilities in the left hemisphere, PMv showed symmetrical connection probabilities. The apparent left-hemisphere lateralization of PMd connectivity provides a potential anatomical substrate for previous reports of left-hemisphere dominance, particularly in PMd, for action selection using learned conditional rules (Rushworth et al., 1998; Schluter et al., 1998, 2001). We also found asymmetries that were specific to particular PM-parieto-frontal pathways. For example, left lateralization of PFdl connections was present for PMd but not PMv, whereas right lateralization of ANG connections was present for PMv but not for PMd. All these apparent asymmetries should be interpreted with caution, because results will be influenced not only by asymmetries in underlying anatomical pathways but also by morphological asymmetries that 
might differentially influence ease of tracking in the two hemispheres.

There are limitations to the information provided by tractography particularly, compared with conventional tract tracing in animal models. As with any imaging modality, results will be limited by data quality and analysis approaches. Here we used high angular resolution diffusion data and a multifiber probabilistic tractography algorithm that can track through regions of fiber crossing or complexity (Behrens et al., 2007). Nevertheless, tractography results are insensitive to small pathways and cannot differentiate anterograde from retrograde connections. Importantly, however, tractography allows us to demonstrate that much of what has been learned of connectional anatomy in the monkey is of relevance for understanding the human brain.

\section{References}

Alkadhi H, Crelier GR, Boendermaker SH, Golay X, Hepp-Reymond MC, Kollias SS (2002a) Reproducibility of primary motor cortex somatotopy under controlled conditions. AJNR Am J Neuroradiol 23:1524-1532.

Alkadhi H, Crelier GR, Boendermaker SH, Hepp-Reymond MC, Kollias SS (2002b) Somatotopy in the ipsilateral primary motor cortex. NeuroReport 13:2065-2070.

Amiez C, Kostopoulos P, Champod AS, Petrides M (2006) Local morphology predicts functional organization of the dorsal premotor region in the human brain. J Neurosci 26:2724-2731.

Anwander A, Tittgemeyer M, von Cramon D, Friederici A, Knosche T (2007) Connectivity-based parcellation of Broca's area. Cereb Cortex 17:816-825.

Aron AR, Poldrack RA (2006) Cortical and subcortical contributions to Stop signal response inhibition: role of the subthalamic nucleus. J Neurosci 26:2424-2433.

Aron AR, Behrens TE, Smith S, Frank MJ, Poldrack RA (2007) Triangulating a cognitive control network using diffusion-weighted magnetic resonance imaging (MRI) and functional MRI. J Neurosci 27:3743-3752.

Astafiev SV, Shulman GL, Stanley CM, Snyder AZ, Van Essen DC, Corbetta M (2003) Functional organization of human intraparietal and frontal cortex for attending, looking, and pointing. J Neurosci 23:4689-4699.

Barbas H, Pandya DN (1987) Architecture and frontal cortical connections of the premotor cortex (area 6) in the rhesus monkey. J Comp Neurol 256:211-228.

Behrens TE, Johansen-Berg H (2005) Relating connectional architecture to grey matter function using diffusion imaging. Philos Trans R Soc Lond B Biol Sci 360:903-911.

Behrens TE, Johansen-Berg H, Woolrich MW, Smith SM, Wheeler-Kingshott CA, Boulby PA, Barker GJ, Sillery EL, Sheehan K, Ciccarelli O, Thompson AJ, Brady JM, Matthews PM (2003a) Non-invasive mapping of connections between human thalamus and cortex using diffusion imaging. Nat Neurosci 6:750-757.

Behrens TE, Woolrich MW, Jenkinson M, Johansen-Berg H, Nunes RG, Clare S, Matthews PM, Brady JM, Smith SM (2003b) Characterization and propagation of uncertainty in diffusion-weighted MR imaging. Magn Reson Med 50:1077-1088.

Behrens TE, Johansen-Berg H, Jbabdi S, Rushworth MF, Woolrich MW (2007) Probabilistic diffusion tractography with multiple fibre orientations: what can we gain? NeuroImage 34:144-155.

Beurze SM, de Lange FP, Toni I, Medendorp WP (2007) Integration of target and effector information in the human brain during reach planning. J Neurophysiol 97:188-199.

Binkofski F, Buccino G, Posse S, Seitz RJ, Rizzolatti G, Freund H (1999a) A fronto-parietal circuit for object manipulation in man: evidence from an fMRI-study. Eur J Neurosci 11:3276-3286.

Binkofski F, Buccino G, Stephan KM, Rizzolatti G, Seitz RJ, Freund HJ (1999b) A parieto-premotor network for object manipulation: evidence from neuroimaging. Exp Brain Res 128:210-213.

Braitenberg V (1962) A note on myeloarchitectonics. J Comp Neurol 118:141-156.

Brett M, Johnsrude IS, Owen AM (2002) The problem of functional localization in the human brain. Nat Rev Neurosci 3:243-249.

Brodmann K (1909) Lokalisationslehre der Grosshirnrinde in ihren Prinzipien dargestellt auf Grund des Zellenbaues. Leipzig: Barth.
Caminiti R, Ferraina S, Johnson PB (1996) The sources of visual information to the primate frontal lobe: a novel role for the superior parietal lobule. Cereb Cortex 6:319-328.

Carmichael ST, Price JL (1995) Sensory and premotor connections of the orbital and medial prefrontal cortex of macaque monkeys. J Comp Neurol 363:642-664.

Catani M, Howard RJ, Pajevic S, Jones DK (2002) Virtual in vivo interactive dissection of white matter fasciculi in the human brain. NeuroImage 17:77-94.

Cavada C, Goldman-Rakic PS (1989) Posterior parietal cortex in rhesus monkey: II. Evidence for segregated corticocortical networks linking sensory and limbic areas with the frontal lobe. J Comp Neurol 287:422-445.

Choi HJ, Zilles K, Mohlberg H, Schleicher A, Fink GR, Armstrong E, Amunts K (2006) Cytoarchitectonic identification and probabilistic mapping of two distinct areas within the anterior ventral bank of the human intraparietal sulcus. J Comp Neurol 495:53-69.

Cipolloni PB, Pandya DN (1999) Cortical connections of the frontoparietal opercular areas in the rhesus monkey. J Comp Neurol 403:431-458.

Croxson PL, Johansen-Berg H, Behrens TE, Robson MD, Pinsk MA, Gross CG, Richter W, Richter MC, Kastner S, Rushworth MF (2005) Quantitative investigation of connections of the prefrontal cortex in the human and macaque using probabilistic diffusion tractography. J Neurosci 25:8854-8866.

de Jong BM, van der Graaf FH, Paans AM (2001) Brain activation related to the representations of external space and body scheme in visuomotor control. NeuroImage 14:1128-1135.

Devlin JT, Sillery EL, Hall DA, Hobden P, Behrens TE, Nunes RG, Clare S, Matthews PM, Moore DR, Johansen-Berg H (2006) Reliable identification of the auditory thalamus using multi-modal structural analyses. NeuroImage 30:1112-1120.

Dum RP, Strick PL (1991) The origin of corticospinal projections from the premotor areas in the frontal lobe. J Neurosci 11:667.

Dum RP, Strick PL (2005) Frontal lobe inputs to the digit representations of the motor areas on the lateral surface of the hemisphere. J Neurosci 25:1375-1386.

Ehrsson HH, Fagergren A, Johansson RS, Forssberg H (2003) Evidence for the involvement of the posterior parietal cortex in coordination of fingertip forces for grasp stability in manipulation. J Neurophysiol 90:2978-2986.

Ehrsson HH, Holmes NP, Passingham RE (2005) Touching a rubber hand: feeling of body ownership is associated with activity in multisensory brain areas. J Neurosci 25:10564-10573.

Eickhoff SB, Heim S, Zilles K, Amunts K (2006) Testing anatomically specified hypotheses in functional imaging using cytoarchitectonic maps. NeuroImage 32:570-582.

Fang PC, Stepniewska I, Kaas JH (2005) Ipsilateral cortical connections of motor, premotor, frontal eye, and posterior parietal fields in a prosimian primate, Otolemur garnetti. J Comp Neurol 490:305-333.

Fogassi L, Gallese V, Buccino G, Craighero L, Fadiga L, Rizzolatti G (2001) Cortical mechanism for the visual guidance of hand grasping movements in the monkey: a reversible inactivation study. Brain 124:571-586.

Friederici AD, Bahlmann J, Heim S, Schubotz RI, Anwander A (2006) The brain differentiates human and non-human grammars: functional localization and structural connectivity. Proc Natl Acad Sci USA 103:2458-2463.

Gallese V, Murata A, Kaseda M, Niki N, Sakata H (1994) Deficit of hand preshaping after muscimol injection in monkey parietal cortex. NeuroReport 5:1525-1529.

Germann J, Robbins S, Halsband U, Petrides M (2005) Precentral sulcal complex of the human brain: morphology and statistical probability maps. J Comp Neurol 493:334-356.

Geyer S (2004) The microstructural border between the motor and the cognitive domain in the human cerebral cortex. Adv Anat Embryol Cell Biol 174: I-VIII, 1-89.

Geyer S, Matelli M, Luppino G, Schleicher A, Jansen Y, Palomero-Gallagher N, Zilles K (1998) Receptor autoradiographic mapping of the mesial motor and premotor cortex of the macaque monkey. J Comp Neurol 397:231-250.

Geyer S, Matelli M, Luppino G, Zilles K (2000) Functional neuroanatomy of the primate isocortical motor system. Anat Embryol 202:443-474.

Graziano MSA (1999) Where is my arm? The relative role of vision and 
proprioception in the neuronal representation of limb position. Proc Natl Acad Sci USA 96:10418-10421.

Grefkes C, Weiss PH, Zilles K, Fink GR (2002) Crossmodal processing of object features in human anterior intraparietal cortex: an fMRI study implies equivalencies between humans and monkeys. Neuron $35: 173-184$.

Grol MJ, de Lange FP, Verstraten FA, Passingham RE, Toni I (2006) Cerebral changes during performance of overlearned arbitrary visuomotor associations. J Neurosci 26:117-125.

Hatanaka N, Tokuno H, Hamada I, Inase M, Ito Y, Imanishi M, Hasegawa N, Akazawa T, Nambu A, Takada M (2003) Thalamocortical and intracortical connections of monkey cingulate motor areas. J Comp Neurol 462:121-138.

He SQ, Dum RP, Strick PL (1993) Topographic organization of corticospinal projections from the frontal lobe: motor areas on the lateral surface of the hemisphere. J Neurosci 13:952.

Heiervang E, Behrens TE, Mackay CE, Robson MD, Johansen-Berg H (2006) Between session reproducibility and between subject variability of diffusion MR and tractography measures. NeuroImage 33:867-877.

Hoshi E, Tanji J (2006) Differential involvement of neurons in the dorsal and ventral premotor cortex during processing of visual signals for action planning. J Neurophysiol 95:3596-3616.

Jeannerod M, Arbib MA, Rizzolatti G, Sakata H (1995) Grasping objects: the cortical mechanisms of visuomotor transformation. Trends Neurosci 18:314-320.

Jenkinson M, Smith SM (2001) Global optimisation for robust affine registration. Med Image Anal 5:143.

Jenkinson M, Bannister P, Brady M, Smith S (2002) Improved optimization for the robust and accurate linear registration and motion correction of brain images. NeuroImage 17:825.

Joachim T (1999) Making large-scale SVM learning practical. In: Advances in Kernel methods-support vector learning (Schölkopf B, Burges, C, Smola A, eds), pp 41-56. Cambridge, MA: MIT.

Johansen-Berg H, Behrens TE, Robson MD, Drobnjak I, Rushworth MFS, Brady JM, Smith SM, Higham DJ, Matthews PM (2004) Changes in connectivity profiles define functionally distinct regions in human medial frontal cortex. Proc Natl Acad Sci USA 101:13335-13340.

Johansen-Berg H, Behrens TE, Sillery E, Ciccarelli O, Thompson AJ, Smith SM, Matthews PM (2005) Functional-anatomical validation and individual variation of diffusion tractography-based segmentation of the human thalamus. Cereb Cortex 15:31-39.

Johnson PB, Ferraina S, Bianchi L, Caminiti R (1996) Cortical networks for visual reaching: physiological and anatomical organization of frontal and parietal lobe arm regions. Cereb Cortex 6:102-119.

Kennerley SW, Walton ME, Behrens TE, Buckley MJ, Rushworth MF (2006) Optimal decision making and the anterior cingulate cortex. Nat Neurosci 9:940-947.

Klein JC, Behrens TE, Robson MD, Mackay CE, Higham DJ, Johansen-Berg H (2007) Connectivity-based parcellation of human cortex using diffusion MRI: establishing reproducibility, validity and observer independence in BA 44/45 and SMA/pre-SMA. NeuroImage 34:204-211.

Kurata K (1994) Information processing for motor control in primate premotor cortex. Behav Brain Res 61:135.

Lewis JW, Van Essen DC (2000) Corticocortical connections of visual, sensorimotor, and multimodal processing areas in the parietal lobe of the macaque monkey. J Comp Neurol 428:112-137.

Lu M-T, Preston JB, Strick PL (1994) Interconnections between the prefrontal cortex and the premotor areas in the frontal lobe. J Comp Neurol 341:375-392.

Luppino G, Murata A, Govoni P, Matelli M (1999) Largely segregated parietofrontal connections linking rostral intraparietal cortex (areas AIP and VIP) and the the ventral premotor cortex (areas F5 and F4). Exp Brain Res 128:181-187.

Luppino G, Rozzi S, Calzavara R, Matelli M (2003) Prefrontal and agranular cingulate projections to the dorsal premotor areas F2 and F7 in the macaque monkey. Eur J Neurosci 17:559-578.

Matelli M, Luppino G, Rizzolatti G (1985) Patterns of cytochrome oxidase activity in the frontal agranular cortex of the macaque monkey. Behav Brain Res 318:125-137.

Matelli M, Camarda R, Glickstein M, Rizzolatti G (1986) Afferent and efferent projections of the inferior area 6 in the macaque monkey. J Comp Neurol 251:281-298.
Matelli M, Govoni P, Galletti C, Kutz DF, Luppino G (1998) Superior area 6 afferents from the superior parietal lobule in the macaque monkey. J Comp Neurol 402:327-352.

Mayka MA, Corcos DM, Leurgans SE, Vaillancourt DE (2006) Threedimensional locations and boundaries of motor and premotor cortices as defined by functional brain imaging: a meta-analysis. NeuroImage 31:1453-1474.

Mori S, Zhang J (2006) Principles of diffusion tensor imaging and its applications to basic neuroscience research. Neuron 51:527-539.

Nakamura H, Kuroda T, Wakita M, Kusunoki M, Kato A, Mikami A, Sakata H, Itoh K (2001) From three-dimensional space vision to prehensile hand movements: the lateral intraparietal area links the hand area v3a and the anterior intraparietal area in macaques. J Neurosci 21:8174-8187.

Passingham RE (1993) The frontal lobes and voluntary action. Oxford: Oxford UP.

Passingham RE, Stephan KE, Kotter R (2002) The anatomical basis of functional localization in the cortex. Nat Rev Neurosci 3:606-616.

Pesaran B, Nelson MJ, Andersen RA (2006) Dorsal premotor neurons encode the relative position of the hand, eye, and goal during reach planning. Neuron 51:125-134.

Petrides M (1985) Deficits in nonspatial conditional associative learning after periarcuate lesions in the monkey. Behav Brain Res 16:95-101.

Preuss TM, Goldman-Rakic PS (1989) Connections of the ventral granular frontal cortex of macaques with perisylvian premotor and somatosensory areas: anatomical evidence for somatic representation in primate frontal association cortex. J Comp Neurol 282:293-316.

Rizzolatti G, Luppino G, Matelli M (1998) The organization of the cortical motor system: new concepts. Electroencephalogr Clin Neurophysiol 106:283.

Rizzolatti G, Fogassi L, Gallese V (2002) Motor and cognitive functions of the ventral premotor cortex. Curr Opin Neurobiol 12:149-154.

Rozzi S, Calzavara R, Belmalih A, Borra E, Gregoriou GG, Matelli M, Luppino G (2006) Cortical connections of the inferior parietal cortical convexity of the macaque monkey. Cereb Cortex 16:1389-1417.

Rushworth MF, Buckley MJ, Gough PM, Alexander IH, Kyriazis D, McDonald KR, Passingham RE (2005) Attentional selection and action selection in the ventral and orbital prefrontal cortex. J Neurosci 25:11628-11636.

Rushworth MFS, Nixon PD, Wade DT, Renowden S, Passingham RE (1998) The left hemisphere and the selection of learned actions. Neuropsychologia 36:11-24.

Rushworth MFS, Behrens T, Johansen-Berg H (2006) Connection patterns distinguish three regions of the human parietal cortex. Cereb Cortex 16:1418-1430.

Schluter ND, Rushworth MFS, Passingham RE, Mills KR (1998) Temporary interference in human lateral premotor cortex suggests dominance for the selection of movements. A study using transcranial magnetic stimulation. Brain 121:785-799.

Schluter ND, Krams M, Rushworth MFS, Passingham RE (2001) Cerebral dominance for action in the human brain: the selection of actions. Neuropsychologia 39:105-113.

Schubotz RI, von Cramon DY (2003) Functional-anatomical concepts of human premotor cortex: evidence from fMRI and PET studies. NeuroImage 20 [Suppl 1]:S120-S131.

Silver MA, Ress D, Heeger DJ (2005) Topographic maps of visual spatial attention in human parietal cortex. J Neurophysiol 94:1358-1371.

Smith SM (2002) Fast robust automated brain extraction. Hum Brain Mapp 17:143.

Stepniewska I, Preuss TM, Kaas JH (1993) Architectonics, somatopic organization, and ipsilateral cortical connections of the primary motor area (M1) of owl monkeys. J Comp Neurol 330:238-271.

Stepniewska I, Preuss TM, Kaas JH (2006) Ipsilateral cortical connections of dorsal and ventral premotor areas in New World owl monkeys. J Comp Neurol 495:691-708.

Takada M, Nambu A, Hatanaka N, Tachibana Y, Miyachi S, Taira M, Inase M (2004) Organization of prefrontal outflow toward frontal motor-related areas in macaque monkeys. Eur J Neurosci 19:3328-3342.

Tanne J, Boussaoud D, Boyer-Zeller N, Rouiller EM (1995) Direct visual pathways for reaching movements in the macaque monkey. NeuroReport 7:267-272.

Tanne-Gariepy J, Rouiller EM, Boussaoud D (2002) Parietal inputs to dor- 
sal versus ventral premotor areas in the macaque monkey: evidence for largely segregated visuomotor pathways. Exp Brain Res 145:91-103.

Toni I, Rushworth MF, Passingham RE (2001) Neural correlates of visuomotor associations. Spatial rules compared with arbitrary rules. Exp Brain Res 141:359-369.

Van Essen DC (1997) A tension-based theory of morphogenesis and compact wiring in the central nervous system. Nature 385:313-318.

Van Hoesen GW, Morecraft RJ, Vogt BA (1993) Connections of the monkey cingulate cortex. In: Neurobiology of cingulate cortex and limbic thalamus (Vogt BA, Gabriel M, eds), pp 249-284. Boston: Birkhauser.

Vogt O, Vogt C (1919) Allgemeinere Ergebnisse unserer Hirnforschung. J Psychol Neurol 25:277.

Watkins KE, Paus T, Lerch JP, Zijdenbos A, Collins DL, Neelin P, Taylor J, Worsley KJ, Evans AC (2001) Structural asymmetries in the human brain: a voxel-based statistical analysis of 142 MRI scans. Cereb Cortex 11:868-877.
Wise SP, di Pellegrino G, Boussaoud D (1996) The premotor cortex and nonstandard sensorimotor mapping. Can J Physiol Pharmacol 74:469.

Wise SP, Boussaoud D, Johnson PB, Caminiti R (1997) Premotor and parietal cortex: corticortical connectivity and combinatorial computations. Annu Rev Neurosci 20:25-42.

Wu CW-H, Bichot NP, Kaas JH (2000) Converging evidence from microstimulation, architecture, and connections for multiple motor areas in the frontal and cingulate cortex of prosimian primates. J Comp Neurol 423:140-177.

Zhang Y, Brady M, Smith S (2001) Segmentation of brain MR images through a hidden Markov random field model and the expectationmaximization algorithm. IEEE Trans Med Imaging 20:45.

Zilles K, Palomero-Gallagher N, Schleicher A (2004) Transmitter receptors and functional anatomy of the cerebral cortex. J Anat 205:417-432. 